\title{
Cochrane
}

Cochrane Database of Systematic Reviews

\section{Self management interventions including action plans for exacerbations versus usual care in people with chronic obstructive pulmonary disease (Protocol)}

Lenferink A, Brusse-Keizer M, van der Valk PDLPM, Frith PA, Zwerink M, Monninkhof EM, van der Palen J, Effing T

Lenferink A, Brusse-Keizer M, van der Valk PDLPM, Frith PA, Zwerink M, Monninkhof EM, van der Palen J, Effing T.

Self management interventions including action plans for exacerbations versus usual care in people with chronic obstructive pulmonary disease.

Cochrane Database of Systematic Reviews 2015, Issue 5. Art. No.: CD011682.

DOI: 10.1002/14651858.CD011682.

www.cochranelibrary.com

Self management interventions including action plans for exacerbations versus usual care in people with chronic obstructive pulmonary disease (Protocol) 
TABLE OF CONTENTS

HEADER . . . . . . . . . . . . . . . . . . . . . . . . . . . . . . . . . . . . 1

ABSTRACT . . . . . . . . . . . . . . . . . . . . . . . . . . . . . . . . . . . . . . . . . .

BACKGROUND . . . . . . . . . . . . . . . . . . . . . . . . . . . . . . . . . . . . .

OBJECTIVES . . . . . . . . . . . . . . . . . . . . . . . . . . . . . . . . . . . . . . .

METHODS . . . . . . . . . . . . . . . . . . . . . . . . . . . . . . . . . . . . . .

ACKNOWLEDGEMENTS . . . . . . . . . . . . . . . . . . . . . . . . . . . . . . . . . . . . . . . . . .

REFERENCES . . . . . . . . . . . . . . . . . . . . . . . . . . . . . . . . . . . . . 7

APPENDICES . . . . . . . . . . . . . . . . . . . . . . . . . . . . . . . . . . . . . 8

CONTRIBUTIONS OF AUTHORS . . . . . . . . . . . . . . . . . . . . . . . . . . . . . . . . . . . . 11

DECLARATIONS OF INTEREST . . . . . . . . . . . . . . . . . . . . . . . . . . . . . . . 11

Self management interventions including action plans for exacerbations versus usual care in people with chronic obstructive pulmonary i disease (Protocol)

Copyright $\odot 2015$ The Cochrane Collaboration. Published by John Wiley \& Sons, Ltd. 


\title{
Self management interventions including action plans for exacerbations versus usual care in people with chronic obstructive pulmonary disease
}

\author{
Anke Lenferink ${ }^{1}$, Marjolein Brusse-Keizer ${ }^{1}$, Paul DLPM van der Valk ${ }^{1}$, Peter A Frith ${ }^{2,3}$, Marlies Zwerink ${ }^{1}$, Evelyn M Monninkhof ${ }^{4}$, \\ Job van der Palen ${ }^{1,5}$, Tanja Effing 3,6 \\ ${ }^{1}$ Department of Pulmonary Medicine, Medische Spectrum Twente, Enschede, Netherlands. ${ }^{2}$ Respiratory Clinical Research Unit, \\ Repatriation General Hospital, Daw Park, Australia. ${ }^{3}$ School of Medicine, Flinders University, Adelaide, Australia. ${ }^{4}$ Julius Center for \\ Health Sciences and Primary Care, University Medical Center Utrecht, Utrecht, Netherlands. ${ }^{5}$ Department of Research Methodology, \\ Measurement, and Data-Analysis, Faculty of Behavioral Sciences, University of Twente, Enschede, Netherlands. ${ }^{6}$ Department of \\ Respiratory Medicine, Repatriation General Hospital, Adelaide, Australia
}

Contact address: Anke Lenferink, Department of Pulmonary Medicine, Medische Spectrum Twente, Enschede, Netherlands. A.Lenferink@mst.nl.

Editorial group: Cochrane Airways Group.

Publication status and date: New, published in Issue 5, 2015.

Citation: Lenferink A, Brusse-Keizer M, van der Valk PDLPM, Frith PA, Zwerink M, Monninkhof EM, van der Palen J, Effing T. Self management interventions including action plans for exacerbations versus usual care in people with chronic obstructive pulmonary disease. Cochrane Database of Systematic Reviews 2015, Issue 5. Art. No.: CD011682. DOI: 10.1002/14651858.CD011682.

Copyright (C) 2015 The Cochrane Collaboration. Published by John Wiley \& Sons, Ltd.

\section{A B S T R A C T}

This is the protocol for a review and there is no abstract. The objectives are as follows:

To evaluate the efficacy of COPD-specific self management interventions that include an action plan for exacerbations of COPD compared with usual care in terms of health-related quality of life, respiratory-related hospital admissions and other health outcomes.

\section{B A C K G R O U N D}

\section{Description of the condition}

Chronic obstructive pulmonary disease (COPD) is characterised by respiratory symptoms that are caused predominantly by persistent airflow limitation, which is usually progressive. It is associated with an enhanced chronic inflammatory response in the lung to noxious particles or gases (Global 2014). Many patients with COPD experience increasing functional impairment and progressive loss of quality of life over many years (Carrasco 2006; Celli
2007; Heyworth 2009). Exacerbations of COPD (ECOPD), defined as acute deteriorations in respiratory health, contribute to functional impairment and risk of mortality in individual patients (Celli 2007; Seemungal 1998). COPD leads to more than six million deaths annually and will be the third leading cause of death worldwide (Global 2014; Lozano 2012). This increased mortality is driven mainly by the expanding global epidemic of smoking, reduced mortality from other common causes of death (e.g. ischaemic heart disease, infectious disease) and increasing age of the world population (Global 2014). Besides mortality, COPD is a leading cause of morbidity. In 2010, COPD was the fifth largest cause of years of life lived with disability (Vos 2012). Apart from

Self management interventions including action plans for exacerbations versus usual care in people with chronic obstructive pulmonary disease (Protocol)

Copyright $\odot 2015$ The Cochrane Collaboration. Published by John Wiley \& Sons, Ltd. 
personal distress, COPD confers a substantial and increasing economic and social burden on society (Global 2014), with its exacerbations accounting for most of the direct costs (Toy 2010).

\section{Description of the intervention}

Self management interventions are defined as structured interventions for individuals aimed at improvement in self health behaviours and self management skills (Lorig 2003). Lorig et al (Lorig 2003) indicated that a self management programme should ideally include training with feedback to improve the following patient skills: problem solving, decision making, resource utilisation, formation of patient-provider partnerships, action planning and self tailoring. Mastery, modelling, interpretation of symptoms and social persuasion skills are believed to contribute to enhanced self efficacy (Lorig 2003). Patients will progressively achieve greater confidence in (self) managing their health, and this will be a powerful factor in inducing new and sustaining behaviours that provide perceived benefit (Bourbeau 2004; Lorig 2003).

Self management has been proposed as an essential part of disease management targeted towards helping patients develop skills to manage a disease more effectively. This is especially important in patients with chronic disease (e.g. COPD, for which the patient is responsible for day-to-day care over the duration of the illness) (Lorig 2003). Self management interventions are associated with reduced duration of exacerbations and hospitalisations and decreased healthcare costs, as well as improved health-related quality of life (HRQoL), for patients with COPD (Effing 2009; Rice 2010; Zwerink 2014). Self management training aims to help patients acquire and improve through practice the skills they need to carry out disease-specific medical regimens (Bourbeau 2009; Effing 2012). It also guides changes in health behaviour and provides emotional support for optimal function of patients with COPD and control of their disease (Bourbeau 2009; Effing 2012). Self management training is considered an increasingly important component of treatment and management of COPD. This training should occur as an interactive and iterative process aimed at sustained behavioural change and instillation of confidence to recognise when an exacerbation is starting and to self manage it effectively and safely (Bourbeau 2009). Self management will not be successful without effective co-operation between patient and healthcare provider (Bodenheimer 2002). Ongoing case manager support is recognised as an additional component required to achieve effective and safe self management (Effing 2012).

Debate on the definition and the most effective content of COPD self management interventions is ongoing (Effing 2012). Recently, an expert group worked on a definition of a COPD self management intervention that is in line with the statements above. When it is released, we will include the definition prepared by this expert group.

Although self management is largely a generic approach, action plans are disease-specific and are considered an intrinsic part of
COPD self management interventions (Effing 2012; Zwerink 2014). Action planning is a frequently applied planning technique adopted to change behaviour (Hagger 2014; Webb 2010). Appropriate use of written COPD action plans enables patients to recognise symptom deterioration early in the course of onset (Walters 2010). Walters et al compared randomised controlled trials (RCTs) of an individual action plan versus RCTs with minimal or no self management education. This review showed that the use of action plans was associated with increased initiation of treatment for acute exacerbations (Walters 2010). However, no evidence suggests that action plans reduced healthcare utilisation, and data on HRQoL were limited (Walters 2010). When integrated into self management programmes, this approach will lead to accelerated initiation of appropriate treatment (Walters 2010) and therefore may reduce exacerbation duration, hospitalisations and healthcare costs for COPD patients without severe comorbidities (Effing 2009; Rice 2010). Appropriate actions can include contacting a healthcare provider for support or initiating self treatment (Wood-Baker 2006). Furthermore, written action plans can include instructions regarding, for example, maintenance treatment.

\section{Why it is important to do this review}

An update of the Cochrane review regarding COPD self management was published in 2014 (Zwerink 2014). This update strengthened the conclusions that self management is associated with improved HRQoL, reduced respiratory-related and all-cause hospitalisations and improved dyspnoea. Subgroup analyses indicate that a standardised exercise component in self management interventions did not change the effects of self management interventions on HRQoL and respiratory-related hospital admissions. However, the review could not reveal the effective components within self management interventions, not least because of heterogeneity among interventions, study populations, follow-up time and outcome measures. Because of the very frequent use of action plans for exacerbations in the included studies, sub-analyses on the use of action plans could not be performed. The contribution of action plans to self-management programmes remains therefore unclear. In the current review, written action plans for ECOPD must be included as part of the self management intervention. This might reduce heterogeneity among interventions, as this review will focus on self treatment interventions that include at least a written action plan for ECOPD.

Since the latest update of the review regarding COPD self management, several studies have been published and new opinions have been raised regarding the limitations and contents of self management interventions with exacerbation action plans for patients with COPD. So far, the evidence regarding COPD action plans is somewhat contradictory. Use of action plans can lead to accelerated initiation of appropriate treatment, thereby reducing the severity of the exacerbation. An RCT conducted by Trappenburg et al showed that early initiation of the agreed upon action plan 
for patients with COPD decreased the impact of exacerbations on health status and tended to accelerate recovery (Trappenburg 2011). However, action plans could also lead to incorrect or delayed treatment when co-morbidities are present. As a result of significantly higher mortality rates in the intervention group, a study evaluating the efficacy of a comprehensive care management programme in reducing the risk for COPD hospitalisation with COPD-specific action plans was prematurely terminated (Fan 2012). This study underlines the need for further investigations of the effectiveness and safety of self management programmes that include self management action plans for patients with COPD. No definitive explanation for these study outcomes has emerged, and they conflict with the positive study outcomes of another highly comparable self management study from Rice et al (Rice 2010). The significantly higher mortality rates in the intervention group reported by Fan et al (Fan 2012) might be explained in part by the use of COPD-specific action plans for patients with COPD and co-morbidities. Findings from a single-centre RCT that included nurse support may provide further insights. In this study, only $42 \%$ of the intervention group could be defined as successful self managers and had significantly reduced risk of hospital readmission (Bucknall 2012). This implies that a significant minority of patients do derive benefit from an intervention with self management components. A study from Bischoff et al showed no long-term benefit in terms of quality of life or improved perceived self efficacy when comprehensive self management or routine monitoring was compared with usual care (Bischoff 2012). However, participants in the self management group seemed more capable of appropriately managing exacerbations than did those in the usual care group (Bischoff 2012). For many COPD self management interventions, action plans are the key component. Nevertheless, these studies show contradictory results. Therefore, in the current review, we will assess the effectiveness of COPD self management interventions that include action plans for ECOPD compared with usual care.

\section{O B J E C T I VES}

To evaluate the efficacy of COPD-specific self management interventions that include an action plan for exacerbations of COPD compared with usual care in terms of health-related quality of life, respiratory-related hospital admissions and other health outcomes.

\section{METHODS}

\section{Criteria for considering studies for this review}

\author{
Types of studies
}

We will include RCTs. We will include studies reported in full text, those published as abstracts only and unpublished data.

\section{Types of participants}

We will include participants with a diagnosis of COPD according to the GOLD (Global Initiative for Chronic Obstructive Lung Disease) classification criteria (Global 2014); those with COPD symptoms (i.e. coughing, sputum or dyspnoea); and participants with a postbronchodilator forced expiratory volume in one second $\left(\mathrm{FEV}_{1}\right)$-to-forced vital capacity $(\mathrm{FVC})$ ratio $<0.70$. We will exclude patients with asthma as a primary diagnosis.

\section{Types of interventions}

We will include trials comparing COPD self management interventions that include a written action plan for ECOPD versus usual care. In this review, an action plan refers to specific behaviour to be initiated when respiratory symptoms deteriorate; it describes when, where and how one should act. An action plan is an agreed upon strategy by which patients will act appropriately when symptoms deteriorate, indicating the start of exacerbations of COPD (e.g. by contacting a healthcare provider for support, by initiating self treatment). It might also include maintenance treatment and advice to avoid situations in which viral infection might be prevalent.

To be included in this review, the self management intervention should include at least formal training on how and when to use an action plan for ECOPD. This formal training programme should be an iterative process between patient and healthcare provider(s) in which feedback is given on the actions. Training should ideally include techniques directed towards behavioural change. In addition, the intervention may include other components that are directed towards behaviour change (e.g. smoking behaviour, exercise or physical activity, diet, use of maintenance medication and correct device use, coping with breathlessness). The intervention content can be delivered to study participants verbally, in writing (hardcopy or digital) or via audiovisual media.

Disease management programmes classified as pulmonary rehabilitation or exercise classes offered in a hospital, at a rehabilitation centre or in a community-based setting will be excluded to avoid as much as possible overlap with pulmonary rehabilitation. Home-based (unsupervised) exercise programmes that include action plans for treatment for exacerbations of COPD will be included, as these studies will ask a more active role of patients and are more clearly aimed at patient self management skills than are supervised exercise programmes.

As the definition, content and focus of COPD self management training in particular, and of COPD treatment in general, have dramatically changed over the past 20 years, we will exclude studies published before 1995 and will search for studies conducted from 1995 until the present date.

Self management interventions including action plans for exacerbations versus usual care in people with chronic obstructive pulmonary 
Types of outcome measures

\section{Primary outcomes}

- Health-related quality of life (HRQoL).

- Respiratory-related hospital admissions.

\section{Secondary outcomes}

- Number of all-cause hospital admissions.

- Use of (other) healthcare facilities (e.g. number of emergency department visits, number of all-cause and respiratory-related hospital admission days in total and per participant, general practitioner, number of nurse and specialist visits).

- Rescue medication use.

- Health status.

- Number of COPD exacerbations.

- Mortality (survival).

- Self efficacy.

- Days lost from work.

Reporting in the trial of one or more of the outcomes listed is not an inclusion criterion for the review. We will divide COPD exacerbations into exacerbations based on COPD symptom scores (e.g. symptom diary), based on courses of oral corticosteroids and based on courses of antibiotics.

\section{Search methods for identification of studies}

\section{Electronic searches}

We will identify trials from the Cochrane Airways Group Specialised Register (CAGR), which is maintained by the Trials Search Co-ordinator for the Group. This Register contains trial reports identified through systematic searches of bibliographic databases including the Cochrane Central Register of Controlled Trials (CENTRAL), MEDLINE, EMBASE, the Cumulative Index to Nursing and Allied Health Literature (CINAHL), the Allied and Complementary Medicine Database (AMED) and PsycINFO, and by handsearching of respiratory journals and meeting abstracts (please see Appendix 1 for further details). We will search all records in the CAGR using the search strategy provided in Appendix 2. We will search from 1995 to the present.

We will also conduct a search of ClinicalTrials.gov (www.ClinicalTrials.gov) and the World Health Organization (WHO) trials portal (www.who.int/ictrp/en/). We will search all databases from their inception to the present, and we will impose no restriction on language of publication.

\section{Searching other resources}

We will check reference lists of all primary studies and review articles for additional references. We will search for additional trials using the ClinicalTrials.gov and WHO International Clinical Trials (ICT) databases.

\section{Data collection and analysis}

\section{Selection of studies}

Two review authors (AL and TE) will independently assess titles and abstracts of all references retrieved. Subsequently, two review authors (AL and TE or MB) will independently review full-text versions of potentially relevant reports, will assess these on eligibility for inclusion and will resolve disagreements by discussion with the third review author. If consensus is not reached, they will consult a third review author. We will contact the authors of included studies to ask for further information, if needed.

\section{Data extraction and management}

Two review authors (AL and TE or MB) will independently assess trial quality and will extract the following data from included studies: relevant outcome measures; sample size; demographics of included participants; disease severity; setting, duration and contents of the intervention and potential effect modifiers. We will use normal data extraction forms and spreadsheets. We will complete a data collection form for study characteristics and outcome data that has been piloted on at least one study in the review.

We will note in the 'Characteristics of included studies' table whether outcome data were reported in a useable way. We will resolve disagreements by reaching consensus or by involving a third person (JP or PV). One review author (AL) will transfer data into the Review Manager (RevMan 2014) file. We will double-check that data have been entered correctly by comparing data presented in the systematic review versus data in the study reports. A second review author (TE) will spot-check study characteristics against the trial report for accuracy.

\section{Assessment of risk of bias in included studies}

We will assess risk of bias according to recommendations outlined in the Cochrane Handbook for Systematic Reviews of Interventions (Higgins 2011) for the following items.

- Random sequence generation.

- Allocation concealment.

- Blinding of participants and personnel.

- Blinding of outcome assessment.

- Incomplete outcome data.

- Selective outcome reporting.

- Other bias. 
For each included study, two review authors (AL and TE or MB) will independently assess for all domains listed above whether high, low or unclear risk of bias is present. Unclear risk indicates that insufficient detail of what happened in the study is reported; that what happened in the study was known but the risk of bias was unknown; or that an entry was not relevant to the study at hand. Each judgement of risk of bias will be supported by a short description of what was reported to have happened in the specific study. The grade of each potential bias from the included study together with a quote from the study report and justification for our judgement will be reported in the 'Risk of bias' table. In case of a cluster-RCT, we will assess risk of recruitment bias, risk of bias for baseline imbalance, risk of bias due to loss of clusters, risk of bias due to incorrect analysis and publication bias. We will resolve disagreements by discussion or with involvement of another review author (JP or PV).

\section{Assesment of bias in conducting the systematic review}

We will conduct the review according to this published protocol and will report deviations form it in the 'Differences between protocol and review' section of the systematic review.

\section{Measures of treatment effect}

We will analyse the results of studies using random-effects modelling in Review Manager (RevMan) 5.3 (RevMan 2014). To compare results across trials, we will use forest plots. We will express the results of each RCT as odds ratios (ORs) with corresponding 95\% confidence intervals (95\% CIs) for dichotomous outcomes, and as mean differences (MDs) or standardised mean differences (SMDs) for continuous outcomes. For primary analyses, we will use the calculator tool in RevMan along with information from adjusted scores (analysis of co-variance (ANCOVA)), change from baseline scores or final scores to create a single forest plot. We will use the calculator tool with the generic inverse variance method for dichotomous or continuous data to allow transformation from data on effect sizes, confidence intervals and standard errors to data required by RevMan to create forest plots with, for example, RRs or mean differences. We will determine the clinical relevance of treatment effects by using the minimal clinically important difference (MCID), when available. We will calculate numbers needed to treat for an additional beneficial outcome (NNTB) for both respiratory-related and all-cause hospital admissions using pooled ORs and control group data from individual studies within the meta-analysis to obtain study-specific NNTB, with Visual Rx 3 (Cates 2008).

\section{Unit of analysis issues}

The unit of analysis in the included RCTs will be the participant. We will also include cluster-RCTs with the cluster as the unit of analysis. For more recent studies, it is most likely that clusters have been taken into account in the analyses. If not, we will adjust for the clusters.

\section{Dealing with missing data}

In cases of missing or incomplete data, we will contact the study authors. If they do not respond, we will make a second attempt to request missing data. If study authors do not respond after a second attempt, we will analyse only the available data and will describe that data are missing. We will list under the heading 'Acknowledgements' study authors who have contributed to this version of the review.

\section{Assessment of heterogeneity}

We will explore variability among studies by performing visual inspection and using the $\mathrm{I}^{2}$ statistic (Higgins 2011). If we identify substantial heterogeneity $\left(\mathrm{I}^{2}>50 \%\right.$ ), we will discuss possible explanations and will critically reconsider the appropriateness of a meta-analysis. In the meta-analysis, we will use a random-effects model (REM), rather than a fixed-effect model (FEM), to account for heterogeneity.

\section{Assessment of reporting biases}

We will explore possible reporting bias by assessing asymmetry in funnel plots to determine whether studies are selectively reported as indicated in the paragraph, 'Assessment of risk of bias in included studies'.

\section{Data synthesis}

When appropriate, we will perform a meta-analysis using RevMan. We will consider a meta-analysis when at least three studies have reported sufficient data for the outcome. Because of the nature of the intervention, we expect to see clinical heterogeneity between studies. If pooling is possible, we will perform meta-analyses using REM rather than FEM.

\section{Summary of findings table}

Using the criteria outlined in the Cochrane Handbook for Systematic Reviews of Interventions (Higgins 2011), we will create a 'Summary of findings' (SOF) table that includes key information concerning the quality of evidence, the magnitude of effect of the self management intervention and the sum of available data on the main outcomes. We will assess potential effect modifiers on participant and self management intervention levels and will structure the SOF table according to these effect modifiers (e.g. exercise, education, health literacy, training of case managers, motivation of the participant, compliance, inclusion and exclusion criteria, individual 
participant data). In the Discussion section, we will assess the potential (modifying) effect of delivery of the intervention. We will use the five GRADE (Grades of Recommendation, Assessment, Development and Evaluation) considerations (study limitations, consistency of effect, imprecision, indirectness and publication bias) to assess the quality of a body of evidence as it relates to studies that contribute data to the meta-analyses for prespecified outcomes. We will use methods and recommendations described in Section 8.5 and Chapter 12 of the Cochrane Handbook for Systematic Reviews of Interventions (Higgins 2011) by using GRADEpro (GRADEpro) software. We will justify all decisions to downgrade or upgrade the quality of studies by using footnotes, and we will provide comments to aid the reader's understanding of the review when necessary.

\section{Subgroup analysis and investigation of heterogeneity}

We will consider subgroup analyses as possible when at least three studies can be included in each subgroup. We intend to perform the following subgroup analyses to explain possible heterogeneity between the results of included studies (if data are available) to determine whether outcomes differ in terms of the following.

- Duration of follow-up: fewer than 12 months of follow-up after the start of the study versus 12 or more months of followup after the start of the study. Shorter-term and longer-term effects of self management interventions including action plans might be different. After follow-up of 12 to 18 months, the effects might be diluted. In addition, we will perform explorative analyses by using different cutoff points for follow-up times (e.g. six months, 18 months).

- Inclusion of participants in the acute phase: inclusion of participants with COPD in the acute unstable phase (with an acute exacerbation of COPD) versus inclusion of participants in the non-acute stable phase (at least four weeks post exacerbation and six weeks post hospitalisation). Acute exacerbations may threaten self management improvements. Awareness of the clinical sequelae of acute exacerbations of COPD enables approaches such as early postexacerbation rehabilitation to mitigate its negative effects (Goldstein 2014).

- Use of a standardised exercise programme as part of the intervention: use of an exercise component in self management versus no exercise component. Increased exercise capacity may result in better HRQoL and potentially fewer hospital admissions.

- Use of a smoking cessation programme in the intervention: smoking cessation component in self management versus no smoking cessation component. Smoking cessation may result in improved HRQoL.

- Self management as part of usual care: low-level usual care versus high-level usual care. Usual care differs significantly between countries and healthcare systems, and sometimes self management will already be included as part of usual care.
Therefore, we will classify according to whether self management was likely to be part of usual care.

We will use the formal test for subgroup interactions in Review Manager (RevMan 2014).

In addition, we will assess the integration of no more than 16 clusters of behavioural change techniques (BCTs) in an explorative subgroup analysis to promote uptake and optimal use of COPDspecific self management behaviour patterns in the intervention. The BCT taxonomy is a methodological tool for specifying intervention content (Michie 2013). The BCT taxonomy (version 1) published by Michie et al (Michie 2013) describes 93 hierarchically clustered techniques in 16 clusters. The BCT must be an observable, replicable and irreducible component of an intervention designed to alter or redirect causal processes that regulate behaviour; that is, a technique that is proposed to be an "active ingredient" (Michie 2011). In this subanalysis, we will classify interventions by their number of BCT taxonomy clusters ('lower or equal' vs 'higher' than the median of BCT clusters found in all included interventions) (Michie 2013). The following 16 BCT clusters included as part of the behavioural change component indicate that the study made an attempt to integrate behavioural change.

- Goals and planning.

- Feedback and monitoring.

- Social support.

- Shaping of knowledge.

- Natural consequences.

- Comparison of behaviours.

- Associations.

- Repetition and substitution.

- Comparison of outcomes.

- Reward and threat.

- Regulation.

- Antecedents.

- Identity.

- Scheduled consequences.

- Self belief.

- Covert learning.

In exploratory analyses, we will assess potential effect modifiers by participant and self management intervention levels (e.g. disease severity, inclusion of participants with co-morbidities, training of case managers, inclusion of an exercise component, behavioural change techniques). This assessment will yield predictors for (more) effective self management programmes. Finally we will try to collect information about the intention of the self management intervention and how it was delivered to participants.

\section{Sensitivity analysis}

We plan to carry out sensitivity analyses under different assumptions to investigate the robustness of effect sizes found in this review. We will undertake sensitivity analyses to identify whether 
review findings are dependent on decisions made during the review process. During the course of the review, we may identify study characteristics for inclusion in the sensitivity analysis. As a risk difference will lead to wide variance in baseline risks, we will use ORs and MDs for the sensitivity analyses. We will also use FEM for the sensitivity analysis.

\section{ACKNOWLEDGEMENTS}

The Background and Methods sections of this protocol are based on a standard template used by the Cochrane Airways Group. Ian Yang served as the Editor for this review and commented critically on the review.

\section{REFERE N CES}

\section{Additional references}

\section{Bischoff 2012}

Bischoff EW, Akkermans R, Bourbeau J, van Weel C, Vercoulen JH, Schermer TR. Comprehensive self management and routine monitoring in chronic obstructive pulmonary disease patients in general practice: randomised controlled trial. BMJ 2012;345:e7642.

Bodenheimer 2002

Bodenheimer T, Lorig K, Holman H, Grumbach K. Patient self-management of chronic disease in primary care. JAMA 2002;288(19):2469-75.

\section{Bourbeau 2004}

Bourbeau J, Nault D, Dang-Tan T. Self-management and behaviour modification in COPD. Patient Education and Counseling 2004;52(3):271-7.

\section{Bourbeau 2009}

Bourbeau J, van der Palen J. Promoting effective selfmanagement programmes to improve COPD. European Respiratory Journal 2009;33(3):461-3.

Bucknall 2012

Bucknall CE, Miller G, Lloyd SM, Cleland J, McCluskey $\mathrm{S}$, Cotton $\mathrm{M}$, et al. Glasgow supported self-management trial (GSuST) for patients with moderate to severe COPD: randomised controlled trial. BMJ 2012;344:e1060.

\section{Carrasco 2006}

Carrasco Garrido P, de Miguel Diez J, Rejas Gutierrez J, Centeno AM, Gobartt Vazquez E, Gil de Miguel A, et al. Negative impact of chronic obstructive pulmonary disease on the health-related quality of life of patients. Results of the EPIDEPOC study. Health and Quality of Life Outcomes 2006;4:31. [DOI: 10.1186/1477-7525-4-31]

Cates 2008 [Computer program]

Cates C. Visual Rx. http://www.nntonline.net/, 2008.
Celli 2007

Celli BR, Barnes PJ. Exacerbations of chronic obstructive pulmonary disease. European Respiratory Journal 2007;29 (6):1224-38.

Effing 2009

Effing T, Kerstjens H, van der Valk PPLDM, Zielhuis G, van der Palen J. (Cost)-effectiveness of self-treatment of exacerbations on the severity of exacerbations in patients with COPD: the COPE II study. Thorax 2009;64(11): 956-62.

Effing 2012

Effing TW, Bourbeau J, Vercoulen J, Apter AJ, Coultas D, Meek P, et al. Self-management programmes for COPD: moving forward. Chronic Respiratory Disease 2012;9(1): $27-35$.

Fan 2012

Fan VS, Gaziano JM, Lew R, Bourbeau J, Adams SG, Leatherman $S$, et al. A comprehensive care management program to prevent chronic obstructive pulmonary disease hospitalizations: a randomized, controlled trial. Annals of Internal Medicine 2012;156(10):673-83.

\section{Global 2014}

Global Initiative for Chronic Obstructive Lung Disease. Global strategy for the diagnosis, management and prevention of chronic obstructive pulmonary disease updated 2014. http://www.goldcopd.org/uploads/users/ files/GOLD_Report2014_Feb07.pdf (accessed 30 January 2015).

\section{Goldstein 2014}

Goldstein R, Brooks D. Pulmonary rehabilitation at the time of the COPD exacerbation. Clinics in Chest Medicine 2014;35(2):391-8.

GRADEpro [Computer program] Brozek J, Oxman A, Schünemann H. GRADEpro. Version 3.2 for Windows. McMaster, 2008. 


\section{Hagger 2014}

Hagger MS, Luszczynska A. Implementation intention and action planning interventions in health contexts: state of the research and proposals for the way forward. Applied Psychology: Health and Well-Being 2014;6(1):1-47.

\section{Heyworth 2009}

Heyworth IT, Hazell ML, Linehan MF, Frank TL. How do common chronic conditions affect health-related quality of life?. British Journal of General Practice 2009;59(568): e353-8.

Higgins 2011

Higgins JPT, Green S (editors). Cochrane Handbook for Systematic Reviews of Interventions Version 5.1 [updated March 2011]. The Cochrane Collaboration, 2011. www.cochrane-handbook.org.

Lorig 2003

Lorig KR, Holman H. Self-management education: history, definition, outcomes, and mechanisms. Annals of Behavioural Medicine 2003;26(1):1-7.

Lozano 2012

Lozano R, Naghavi M, Foreman K, Lim S, Shibuya K, Aboyans V, et al. Global and regional mortality from 235 causes of death for 20 age groups in 1990 and 2010: a systematic analysis for the Global Burden of Disease Study 2010. Lancet 2012;380(9859):2095-128.

\section{Michie 2011}

Michie S, Abraham C, Eccles MP, Francis JJ, Hardeman W, Johnston M. Strengthening evaluation and implementation by specifying components of behaviour change interventions: a study protocol. Implementation Science 2011;10:6.

Michie 2013

Michie S, Richardson M, Johnston M, Abraham C, Francis J, Hardeman W, et al. The behavior change technique taxonomy (v1) of 93 hierarchically clustered techniques: building an international consensus for the reporting of behavior change interventions. Annals of Behavioral Medicine 2013;46(1):81-95.

RevMan 2014 [Computer program]

The Nordic Cochrane Centre, The Cochrane Collaboration. Review Manager (RevMan). Version 5.3. Copenhagen: The Nordic Cochrane Centre, The Cochrane Collaboration, 2014.

Rice 2010

Rice KL, Dewan N, Bloomfield HE, Grill J, Schult TM, Nelson DB, et al. Disease management program for chronic obstructive pulmonary disease: a randomized controlled trial. American Journal of Respiratory and Critical Care

Medicine 2010;182(7):890-6.

\section{Seemungal 1998}

Seemungal TA, Donaldson GC, Paul EA, Bestall JC, Jeffries DJ, Wedzicha JA. Effect of exacerbation on quality of life in patients with chronic obstructive pulmonary disease. American Journal of Respiratory and Critical Care Medicine 1998;157(5 pt 1):1418-22.

Toy 2010

Toy EL, Gallagher KF, Stanley EL, Swensen AR, Duh MS. The economic impact of exacerbations of chronic obstructive pulmonary disease and exacerbation definition: a review. COPD 2010;7(3):214-28.

\section{Trappenburg 2011}

Trappenburg JC, Monninkhof EM, Bourbeau J, Troosters T, Schrijvers AJ, Verheij TJ, et al. Effect of an action plan with ongoing support by a case manager on exacerbationrelated outcome in patients with COPD: a multicentre randomised controlled trial. Thorax 2011;66(11):977-84.

Vos 2012

Vos T, Flaxman AD, Naghavi M, Lozano R, Michaud C, Ezzati M, et al. Years lived with disability (YLDs) for 1160 sequelae of 289 diseases and injuries 1990-2010: a systematic analysis for the Global Burden of Disease Study 2010. Lancet 2012; Vol. 380, issue 9859:2163-96.

\section{Walters 2010}

Walters JA, Turnock AC, Walters EH, Wood-Baker R. Action plans with limited patient education only for exacerbations of chronic obstructive pulmonary disease. Cochrane Database of Systematic Reviews 2010, Issue 5. [DOI: 10.1002/14651858.CD005074.pub3]

\section{Webb 2010}

Webb TL, Sniehotta FF, Michie S. Using theories of behaviour change to inform interventions for addictive behaviours. Addiction 2010;105(11):1879-92.

\section{Wood-Baker 2006}

Wood-Baker R, McGlone S, Venn A, Walters EH. Written action plans in chronic obstructive pulmonary disease increase appropriate treatment for acute exacerbations. Respirology 2006;11(5):619-26.

\section{Zwerink 2014}

Zwerink M, Brusse-Keizer M, van der Valk PD, Zielhuis GA Monninkhof EM, van der Palen J, et al. Self management for patients with chronic obstructive pulmonary disease. Cochrane Database of Systematic Reviews 2014, Issue 3. [DOI: 10.1002/14651858.CD002990.pub3]

* Indicates the major publication for the study 
A P PENDICES

\section{Appendix I. Sources and search methods for the Cochrane Airways Group Specialised Register (CAGR)}

\section{Electronic searches: core databases}

\begin{tabular}{ll}
\hline Database & Frequency of search \\
\hline MEDLINE (Ovid) & Weekly \\
\hline EMBASE (Ovid) & Weekly \\
\hline CENTRAL & Monthly \\
\hline PsycINFO (Ovid) & Monthly \\
\hline CINAHL (EBSCO) & Monthly \\
\hline AMED (EBSCO) & Monthly \\
\hline
\end{tabular}

\section{Handsearches: core respiratory conference abstracts}

\begin{tabular}{l|l}
\hline Conference & Years searched \\
\hline American Academy of Allergy, Asthma and Immunology (AAAAI) & 2001 onwards \\
\hline American Thoracic Society (ATS) & 2001 onwards \\
\hline Asia Pacific Society of Respirology (APSR) & 2004 onwards \\
\hline British Thoracic Society Winter Meeting (BTS) & 2000 onwards \\
\hline Chest Meeting & 2003 onwards \\
\hline European Respiratory Society (ERS) & $1992,1994,2000$ onwards \\
\hline International Primary Care Respiratory Group Congress (IPCRG) & 2002 onwards \\
\hline Thoracic Society of Australia and New Zealand (TSANZ) & 1999 onwards \\
\hline
\end{tabular}

Self management interventions including action plans for exacerbations versus usual care in people with chronic obstructive pulmonary disease (Protocol)

Copyright $\odot 2015$ The Cochrane Collaboration. Published by John Wiley \& Sons, Ltd. 


\section{MEDLINE search strategy used to identify trials for the CAGR}

COPD search

1. Lung Diseases, Obstructive/

2. exp Pulmonary Disease, Chronic Obstructive/

3. emphysema $\$$.mp.

4. (chronic\$ adj3 bronchiti\$).mp.

5. (obstruct $\$$ adj3 (pulmonary or lung $\$$ or airway $\$$ or airfl ow $\$$ or bronch $\$$ or respirat $\$$ )).mp.

6. COPD.mp.

7. COAD.mp.

8. COBD.mp.

9. AECB.mp.

10. or/1-9

\section{Filter to identify RCTs}

1. exp "clinical trial [publication type]"/

2. (randomised or randomised).ab,ti.

3. placebo.ab,ti.

4. dt.fs.

5. randomly.ab,ti.

6. trial.ab,ti.

7. groups.ab,ti.

8. or/1-7

9. Animals/

10. Humans/

11. 9 not (9 and 10)

12. 8 not 11

The MEDLINE strategy and RCT lter are adapted to identify trials in other electronic databases.

\section{Appendix 2. Search strategy to identify relevant trials from the CAGR}

Proposed search strategy for Airways Register

\#1 MeSH DESCRIPTOR Pulmonary Disease, Chronic Obstructive Explode All

\#2 MeSH DESCRIPTOR Bronchitis, Chronic

\#3 (obstruct*) near3 (pulmonary or lung* or airway* or airflow* or bronch* or respirat*)

\#4 COPD:MISC1

\#5 (COPD OR COAD OR COBD):TI,AB,KW

\#6 \#1 OR \#2 OR \#3 OR \#4 OR \#5

\#7 MeSH DESCRIPTOR Self Care Explode All

\#8 MeSH DESCRIPTOR Education

\#9 MeSH DESCRIPTOR Patient Education as Topic

\#10 educat*

\#11 self-manag*

\#12 "self manag*"

\#13 self-car* or "self car*"

\#14 train* or instruct*

\#15 "patient cent*" or patient-cent*

\#16 patient-focus* or "patient focus*"

\#17 patient-education or "patient education"

\#18 "management plan" or management-plan

\#19 management* NEAR1 program*

\#20 behavior* or behaviour*

Self management interventions including action plans for exacerbations versus usual care in people with chronic obstructive pulmonary 
\#21 disease* NEAR2 management*

\#22 self-efficac*

\#23 empower*

$\# 24$ action-plan*

\#25 action NEXT plan*

$\# 26 \# 7$ or \#8 or \#9 or \#10 or \#11 or \#12 or \#13 or \#14 or \#15 or \#16 or \#17 or \#18 or \#19 or \#20 or \#21 or \#22 or \#23 or \#24 or \#25

\#27 \#6 AND \#26

\#28 (\#27) AND (INREGISTER)

[Note: in search line \#4, MISC2 denotes the field in which the reference has been coded for condition, in this case, COPD]

\section{CONTRIBUTIONSOFAUTHORS}

Anke Lenferink co-ordinated the review and wrote the protocol. Tanja Effing helped to write the protocol. All other review authors provided critical comments on the protocol.

\section{DECLARATIONSOF INTEREST}

The review authors declare that they received no funding for this systematic review. 